\title{
Reliability Evaluation of Mobile Ad-hoc Networks
}

\author{
Anil Choudhary, O. P. Roy and T. Tuithung \\ North Eastern Regional Institute of Science and Technology ("NERIST" Deemed \\ University under MHRD, Govt. of India), Itanagar, (Arunachal Pradesh)
}

\begin{abstract}
Reliability is the important concern in wireless ad hoc networks. Therefore, evaluating reliability has become the essential in MANET. Many existing wireless ad hoc network routing protocols assume the availability of reliable nodes. This, however, is not a realistic assumption given the dynamics of wireless ad hoc networks. Also, due to frequent changes in topology, link failures and node failures are possible. Therefore, a realistic method is needed to evaluate reliability of ad-hoc networks in case of such uncertainties. In this paper, we propose a node failure model, which is injected into the MANET. We then illustrate the effect of node failure on the performance of the mobile ad-hoc networks. This paper evaluates how well a DSR protocols for MANETs behave when under a realistic scenario of momentary node failures and topology changes, which is represented by a node failure model. A gap analysis is then carried out and strategies are planned to improve the performance and reliability of MANET.
\end{abstract}

Keywords: Mobile Ad-hoc Networks (MANET), Constant Hazard Model, Node Failure Model, DSR (Dynamic Source Routing)

\section{ACRONYM}

$\begin{array}{ll}\text { MANET } & \text { Mobile Ad-hoc Networks } \\ \text { CHM } & \text { Constant Hazard Model } \\ \text { DSR } & \text { Dynamic Source Routing } \\ \text { MTTF } & \text { Mean Time to Failure } \\ \text { MTBF } & \text { Mean Time between Failures } \\ \text { NetSim } & \text { Network Simulator }\end{array}$

\section{NOTATIONS}

\section{$\mathbf{z}(\mathbf{t}) \quad$ Hazard Rate Function}

\section{Introduction}

Computing system reliability is an interesting, but difficult, research area. Although there are many reliability models suggested and studied in the literature, none can be used universally, and there is no unique model which can perform well in all situations. The reasons for this is that the assumptions made for each model are correct or are good approximations of the reality only in specific cases[1].

In MANET communication between nodes is done through the wireless medium. Because nodes are mobile and may join or leave the network, MANETs have a dynamic topology. Nodes that are in transmission range of each other are called neighbors. Neighbors can send directly to each other. However, when a node needs to send data to another non-neighboring node, the data is routed through a sequence of multiple hops, with intermediate nodes acting as routers. There are numerous issues to consider when deploying MANETs. The following are some of the main issues [2]. 
- Unpredictability of environment: Ad hoc networks may be deployed in unknown terrains, hazardous conditions, and even hostile environments where tampering or the actual destruction of a node may be imminent. Depending on the environment, node failures may occur frequently.

- Unreliability of wireless medium: Communication through the wireless medium is unreliable and subject to errors. Also, due to varying environmental conditions such as high levels of electro-magnetic interference (EMI) or inclement weather, the quality of the wireless link may be unpredictable. Furthermore, in some applications, nodes may be resource-constrained and thus would not be able to support transport protocols necessary to ensure reliable communication on a lossy link. Thus, link quality may fluctuate in a MANET.

- Resource-constrained nodes: Nodes in a MANET are typically battery powered as well as limited in storage and processing capabilities. Moreover, they may be situated in areas where it is not possible to re-charge and thus have limited lifetimes. Because of these limitations, they must have algorithms which are energy-efficient as well as operating with limited processing and memory resources. The available bandwidth of the wireless medium may also be limited because nodes may not be able to sacrifice the energy consumed by operating at full link speed.

- Dynamic topology: The topology in an ad-hoc network may change constantly due to the mobility of nodes. As nodes move in and out of range of each other, some links break while new links between nodes are created.

As a result of these issues, MANETs are prone to following numerous realistic scenarios of Physical faults of the end to end communication connection [2-3].

- Transmission errors: The unreliability of the wireless medium and the unpredictability of the environment may lead to transmitted packets being garbled and thus received in error.

- Node failures: Nodes may fail at any time due to different types of hazardous conditions in the environment. They may also drop out of the network either voluntarily or when their energy supply is depleted.

- Link failures: Node failures as well as changing environmental conditions (e.g., increased levels of EMI) may cause links between nodes to break.

- Route breakages: When the network topology changes due to node/link failures and/or node/link additions to the network, routes become out-of date and thus incorrect. Depending upon the network transport protocol, packets forwarded through stale routes may either eventually be dropped or be delayed; packets may take a circuitous route before eventually arriving at the destination node.

- Congested nodes or links: Due to the topology of the network and the nature of the routing protocol, certain nodes or links may become over utilized, i.e., congested. This will lead to either larger delays or packet loss.

- Power faults: Power faults are caused by the limited battery life in mobile stations. A router may be incapable of performing the routing task either due to insufficient power, or due to its desire to reserve energy for later use.

Routing protocols for MANETs must deal with these issues to be effective.

\section{A. Approaches used to deal with failures.}

Many algorithms, models, and protocols have been presented to solve network reliability problems [4]-[29]. The failure detection and recovery technique [4] recovers the cluster structure in less than one-fourth of the time taken by the Gupta algorithm and is also proven to be $70 \%$ more energy-efficient than the same. The cluster-based failure detection and recovery scheme [4] proves to be an efficient and quick solution to robust and scalable sensor network for long and sustained operation. 
A novel Workload-aware Reliability evaluation model is proposed in [5], describes the workload grid resources and evaluate the reliability of jobs with constrain to deadline, a new type of resource fault (Deadline-Miss fault) is used in the model.

The effect of nodes failure rates, fault, and the mobility pattern on the reliability are discussed in [6-7, 10, 18-19,25-26].

To deal with failures most commonly used models and protocols are based on multicast [8,27], multi-path [9, 11-12, 16], multi-hop [13], and many-cast [14] routing.

A clustering based on dominating set and spanning tree is also an important approach in MANETs. There are numerous algorithms on this topic, namely, graph theoretic algorithms for clustering in MANETs [15]. Clustering using dominating sets in a MANET was favorable in a medium size and medium speed network in terms of cluster quality, time and message complexities. Spanning tree based approach provided favorable results for a medium size MANET with better run time performance than the dominating set based algorithm. Fully distributed linked cluster architecture is introduced [16] mainly for hierarchical routing and to demonstrate the adaptability of the network to connectivity changes. With the advent of multimedia communications, the use of the cluster architecture for ad hoc network has been revisited by Gerla [17].

Position-based routing algorithms [20] promise to eliminate some of the limitations of topology-based routing by using additional information. The routing decision is then based on the destination's position contained in the packet and the position of the forwarding node's neighbors.

A set of algorithms that address the unpredictable and unreliable behavior exhibited by wireless ad hoc networks are presented in [21]. These new algorithms, together with the new techniques and abstractions introduced in [21], simplify the design of algorithms for wireless ad hoc networks. These abstractions and the algorithms that implement them are referred as virtual infrastructure. Virtual infrastructure (VI) replaces the fixed infrastructure found in more traditional networks, thus compensating for unpredictable and unreliable behavior. A novel routing protocol [22] for wireless, mobile ad-hoc networks incorporates features that enhance routing reliability, defined as the ability to provide almost $100 \%$ packet delivery rate. The protocol is based on a virtual structure, unrelated to the physical network topology, where mobile nodes are connected by virtual links and are responsible for keeping physical routes to their neighbors in the virtual structure.

Architecture to form a grid over a mobile ad hoc network by using trace files that capture the regularity in the movement or rather the stability of the nodes [23]. It has also shown the feasibility of sharing the resources using such a grid by using a theoretical model and simulation studies. Similarly, HarpiaGrid [24], a geography-aware grid-based routing protocol for VANETs (vehicular networks) uses map data to generate a shortest transmission grid route, effectively trades route discovery communication overhead. Rather than using blinding, it reduces unnecessary transmissions by limiting the greedy search in the transmission path, thus greatly improving routing efficiency.

A distributed system-level fault diagnosis algorithm for detecting and diagnosing faulty processors in Dynamic Positioning System (DPS) is introduced in [28]. A new approach to the diagnosis problem, illustrates the procedure of diagnosis verification, which adopts a method of fault injection by setting some faults in the system by programming, and provides the basic idea, the detailed execution steps and the corresponding results. Two implementation models of algorithm are presented in [28], in the first one, network topology doesn't change during diagnosis and both hard and soft faults can be easily detected. In the second model the system topology change is allowed during diagnosis, in this case the ability of diagnosis decreases but it can be rectified too by setting up a fixed area for mobility of nodes.

Studies are carried out for two core fault-tolerant primitives, reliable dissemination and consensus, and presents two families of protocols which implement these primitives in a 
wide range of mobile ad-hoc networks [29]. Simulation studies indicating that they are able to provide their guarantees in a bandwidth efficient manner. This is achieved by taking advantage of the broadcast nature and variable message delivery latencies inherent in ad-hoc networks. To illustrate the usefulness of these two primitives, a design for a distributed, fault-tolerant tuple space suitable to implement on mobile ad-hoc networks is presented in [29].

A survey of recent research publications on performance evaluation of networks were used to show that the majority of results of simulation studies of wireless networks published in technical literature have many pitfalls/issues [30]. These pitfalls and some important issues with some recommendations to increase the reliability and repeatedly of simulation studies are documented in [30].

\section{B. Necessity of the Model}

Evaluating and quantifying reliability is of critical importance in wireless ad hoc networks. Many existing optimization algorithms assume the availability of precise reliability information, which is unrealistic due to the dynamics of ad-hoc networks [31]. Therefore, we present a node failure reliability estimation model that accounts for uncertainty, and the failures.

In constant hazard model [32], the hazard rate function does not change with time. The constant hazard model can be expressed in the form $\mathrm{z}(\mathrm{t})=\lambda$

Where $\lambda$ is a constant, independent of time. Many products, particularly electronic components, exhibit this characteristic. This is the simplest hazard rate model and therefore has been used for many years in the reliability analysis and prediction. Therefore, we have proposed to use constant hazarded model described in [32] for reliability analysis and prediction of MANET.

In the view of realistic scenarios, we have designed a node failure model, which is based on constant hazard model.

In this paper, we have presented

- Design of node failure model using Constant hazard model [32].

- Modeling and reliability evaluation of ad-hoc networks by simulation technology.

- Effect of node failure model on reliability of MANET.

\section{Node Failure Model Design}

Failure rate is the frequency with which an engineered system or component fails, expressed, for example, in failures per hour. It is often denoted by the Greek letter $\lambda$ (lambda) and is important in reliability engineering.

The failure rate of a system usually depends on time, with the rate varying over the life cycle of the system. In practice, the mean time between failures (MTBF, $1 / \lambda$ ) is often reported instead of the failure rate. This is valid and useful if the failure rate may be assumed constant - often used for complex electronics units or systems and is a general agreement in some reliability standards. It does in this case only relate to the flat region of the bathtub curve, also called the "useful life period". Because of this, it is incorrect to extrapolate MTBF to give an estimate of the service life time of a component, which will typically be much less than suggested by the MTBF due to the much higher failure rates in the "end-of-life wearout" part of the "bathtub curve".

The reason for the preferred use for MTBF numbers is that the use of large positive numbers (such as 2000 hours) is more intuitive and easier to remember than very small numbers (such as 0.0005 per hour).

The MTBF is an important system parameter in systems where failure rate needs to be managed, in particular for safety systems. The MTBF appears frequently in the 
engineering design requirements, and governs frequency of required system maintenance and inspections. In special processes called renewal processes, where the time to recover from failure can be neglected and the likelihood of failure remains constant with respect to time, the failure rate is simply the multiplicative inverse of the MTBF $(1 / \lambda)$.

A similar ratio used in the transport industries, especially in railways and trucking is "mean distance between failures", a variation which attempts to correlate actual loaded distances to similar reliability needs and practices.

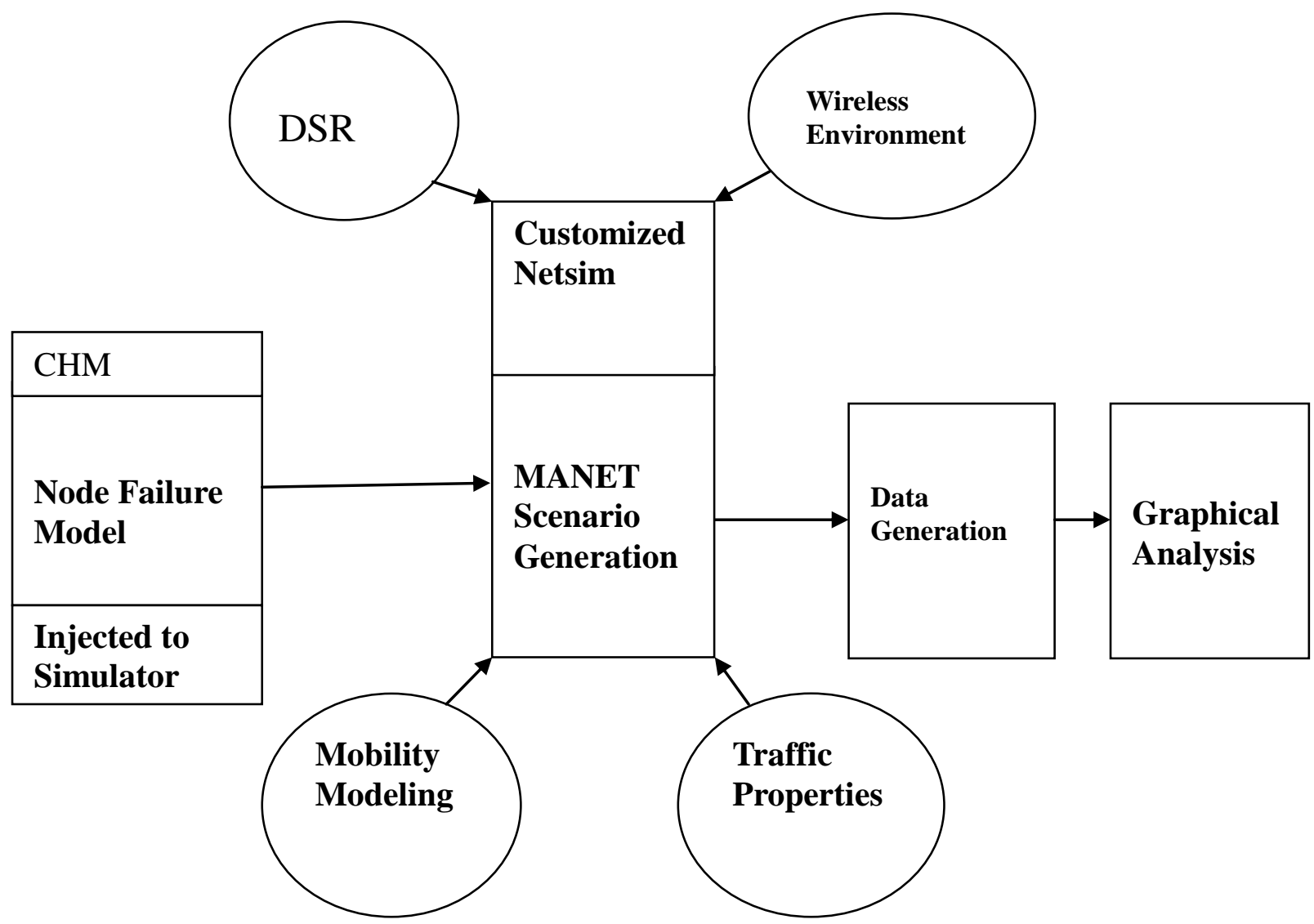

Figure 1. Node Failure Model and Scenario Generation using NetSim

Failure rates are important factors in the insurance, finance, commerce and regulatory industries and fundamental to the design of safe systems in a wide variety of applications.

The node failure model is designed to observe the effect of node failure on the performance and reliability of the network.

\section{Constant Hazard Model}

In constant hazard model [32], the hazard rate function does not change with time. The constant hazard model can be expressed in the form $\mathrm{z}(\mathrm{t})=\lambda$

Where $\lambda$ is a constant, independent of time. Many products, particularly electronic components, exhibit this characteristic.

This is the simplest hazard rate model and therefore has been used for many years in the reliability analysis and prediction. Therefore, we have proposed to use constant hazarded model described in [32] for reliability analysis and prediction of MANET.

In this model, the number of iterations is obtained by dividing total time with granularity. For each iteration the probability is calculated using lamda (1/MTTF) and granularity values has shown below: 
Probability $=1-\exp (-$ lamda $x$ Granularity $)$

A random number is generated for each node in a particular iteration. A node is failed if random number generated is less than the probability value. This procedure is repeated for all the iterations.

\section{Pseudo code of Constant Hazard Model}

iterations $=$ total time/granularity

for each iteration do \{

calculate probability using nlamda and granularity

for each node do\{

$$
\begin{aligned}
& \text { if node is failed already\{ } \\
& \text { go to next node }
\end{aligned}
$$

Generate random number

if random number is less than probability \{

fail the node 


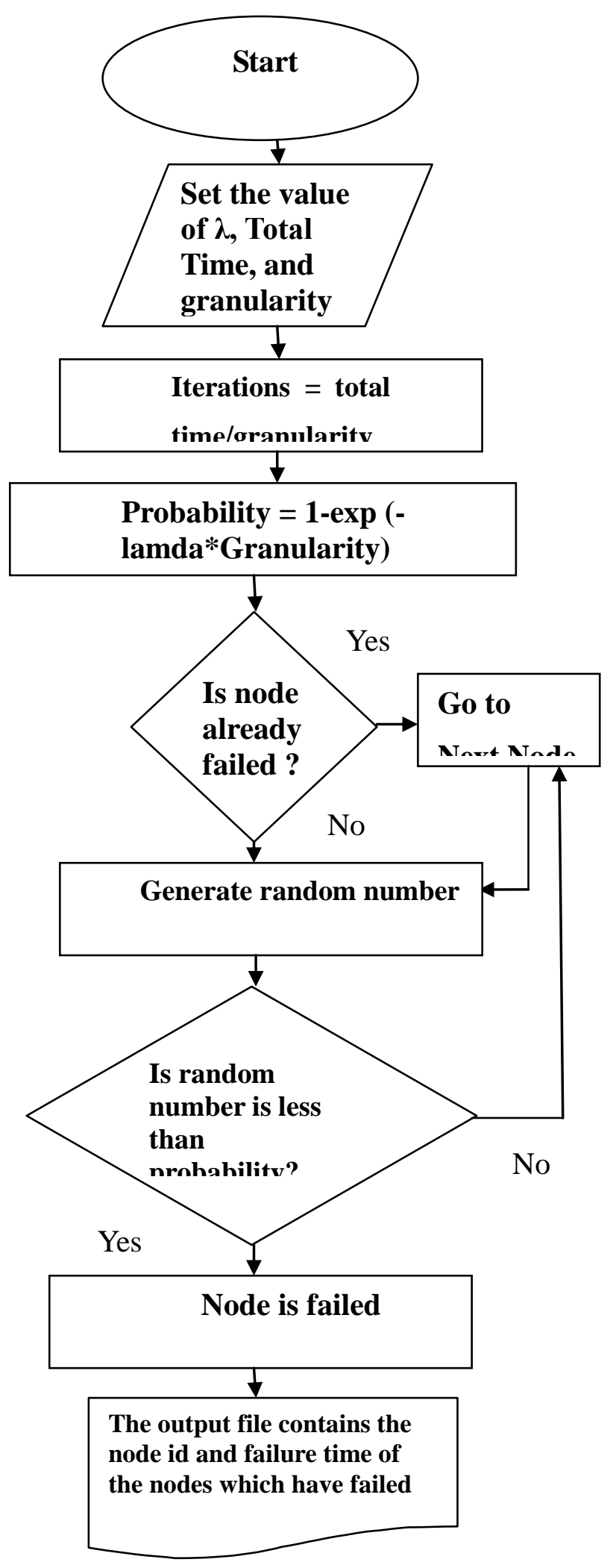

Figure 2. Flow Chart for Node Failure Model

The output file contains the node id and failure time of the nodes which have failed. 


\section{Customizing NetSim}

We have developed custom code in NetSim using VC++. In this section, Visual Studio 2005 is used to create the dlls. We have modified the primitives of the protocol as per our requirement and set the properties for the simulation scenarios.

\section{Source Code Modifications to NetSim}

The constant hazard hard model outputs a file which indicates the time at which various nodes fail. Using this as an input, to NetSim we made the following modifications to the source code to fail nodes

\section{How to fail a Node in NetSim}

- Using Device Id: Identify the Device ID of the particular device.

- The fn_NetSim_DSR_Run()is the main function to handle all the protocol functionalities as per the logic the device which is satisfying the below condition gets failured.

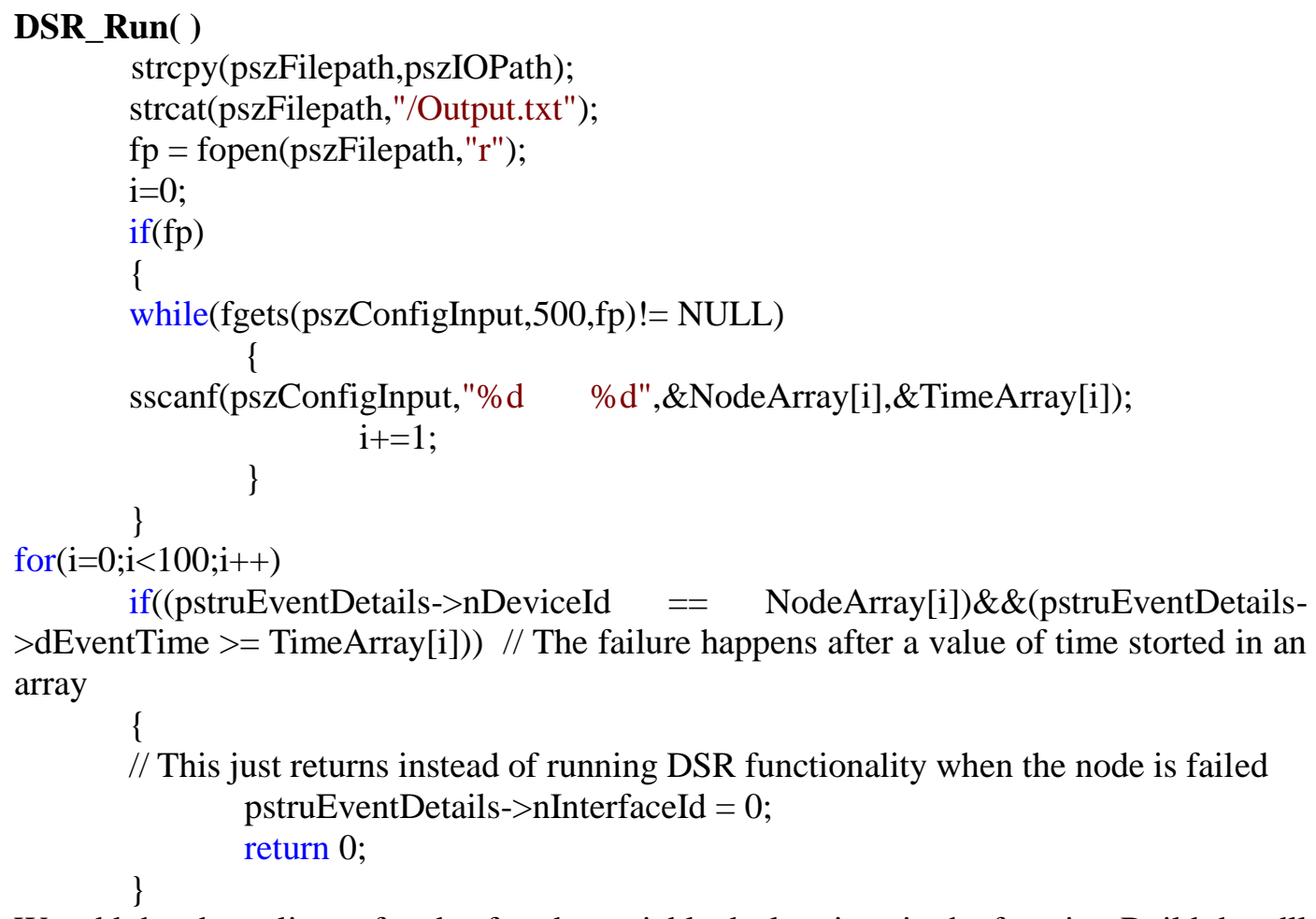

We add the above lines of code after the variable declarations in the function Build the .dll by clicking Build $\rightarrow$ Build Solution. (The dll will be created inside the Visual studio project $\rightarrow$ Debug)

\section{MANET Simulation Scenarios}

We have setup simulation scenarios and designed simulation model for reliability analysis of Ad Hoc network. We have used NetSim Standard Version 6 - network simulator for simulations.

We preferred NetSim due to following advantages:

- In-built development environment.

- Protocol libraries with open source code. 
- Technically reviewed for scientific correctness.

Since, routing is one of the important aspects of ad-hoc networks towards reliability evaluation; we are considering DSR protocols for analysis using NetSim ${ }^{\text {TM }}$-Network Simulation Software developed by Tetcos, Bangalore, India.

The NetSim is customized for incorporating node failure model.

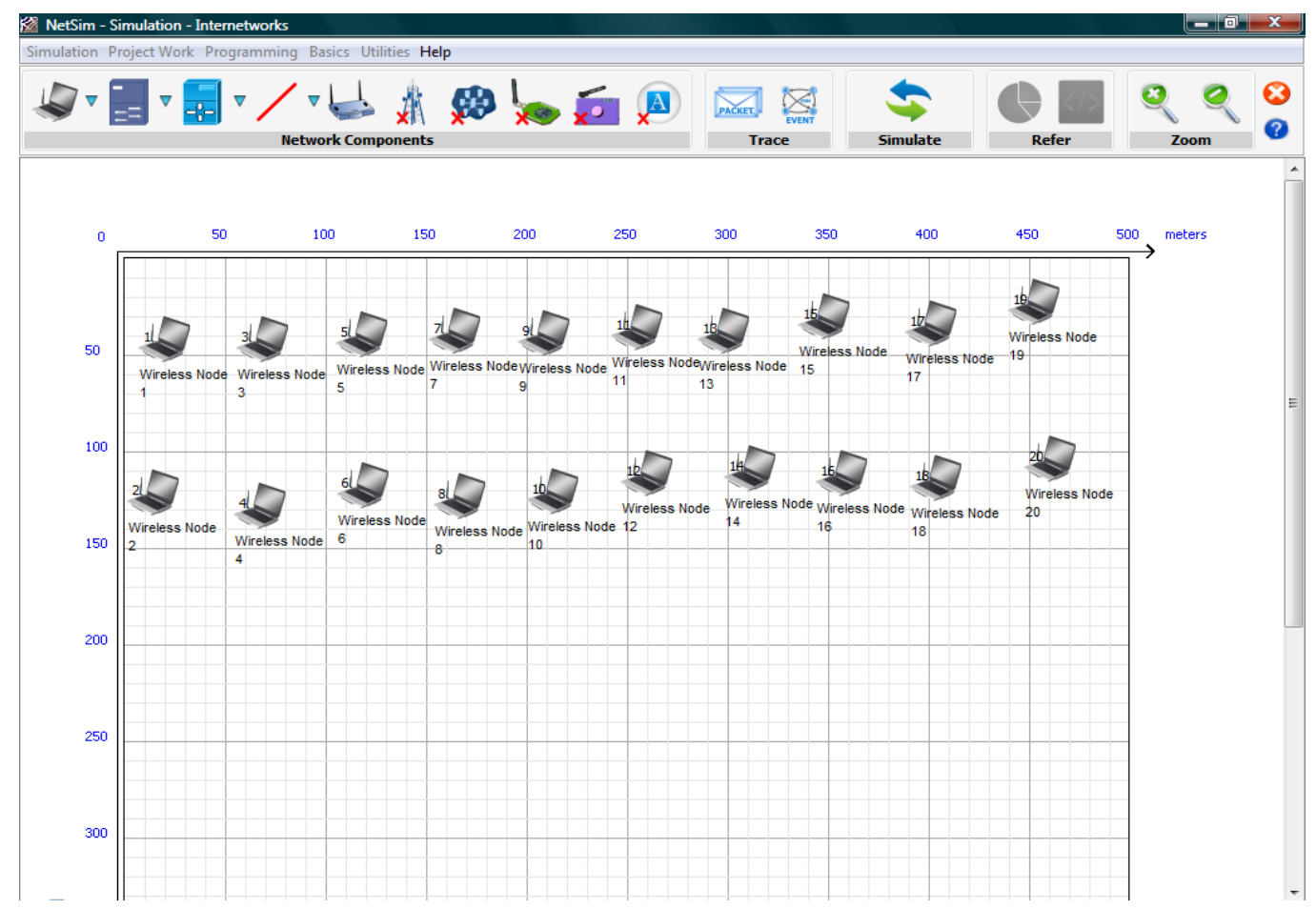

Figure 3. Snap Shot of Simulation Scnario

Table 1. Effect of Number of Failed Nodes on Reliability

\begin{tabular}{|c|c|c|c|c|c|}
\hline $\begin{array}{c}\text { Lamda in per } \\
\text { second }\end{array}$ & $\begin{array}{c}\text { MTTF in } \\
\text { seconds }\end{array}$ & Granularity(s) & $\begin{array}{c}\text { Number } \\
\text { of } \\
\text { Failed } \\
\text { nodes }\end{array}$ & $\begin{array}{c}\text { Throughput } \\
\text { (Mbps) }\end{array}$ & Reliability \\
\hline 0.0001 & 10000 & 10 & 0 & 0.406035 & 0.999 \\
\hline 0.0002 & 5000 & 10 & 2 & 0.365365 & 0.998 \\
\hline 0.001 & 1000 & 10 & 4 & 0.316744 & 0.99 \\
\hline 0.01 & 100 & 10 & 12 & 0.253832 & 0.904 \\
\hline 0.013333333 & 75 & 10 & 14 & 0.233921 & 0.875 \\
\hline 0.02 & 50 & 10 & 17 & 0.17498 & 0.8187 \\
\hline 0.033333333 & 30 & 10 & 20 & 0.135658 & 0.716 \\
\hline 0.05 & 20 & 10 & 20 & 0.10842 & 0.607 \\
\hline 0.1 & 10 & 10 & 20 & 0.046982 & 0.38 \\
\hline
\end{tabular}




\section{Traffic Properties}

- Source: Node N

- Destination: Node N+1

- This means, Node 1 to Node 2, Node 2 to Node 3 and so on and finally Node 20 to Node 1

- Transmission Type: Point to Point

- Traffic Type: Custom

- Application Data size: 512 Bytes, Constant distribution

- Inter-arrival time: 200,000 micro seconds, constant distribution

Mobility model properties are same for all the nodes

- Mobility Model: Random Way point

- Pause Time: 1 second

- Velocity: $5 \mathrm{~m} / \mathrm{sec}$

\section{Wireless Environment Properties:}

Fading_figure $=0$

Frequency $=2412$

Path_loss_exponent=3

Standard_deviation $=0$

\section{Simulation Time - 100 Sec}

In this experiment, nodes are failed using constant hazard mode where MTTF is given by (1/lamda) with lamda being the failure rate. Simulation time is divided into intervals using granularity. The number of intervals is obtained

by dividing simulation time by granularity. In each interval the probability that a node can fail is given by

$\mathrm{P}$ (node failure $)=1-\mathrm{e}^{(-\lambda \times \mathrm{g})}$ where $\lambda$ is the failure rate and $\mathrm{g}$ is the granularity.

In this experiment, simulation time is 100 seconds and Granularity is 10 seconds. MTTF is varied from 10 seconds to 10000 seconds as shown in the graph and the network is simulated. The throughput is obtained for each value of MTTF.

\section{Results and Analysis}

From the figure it can be observed that throughput decreases as MTTF decreases. As MTTF decreases lamda increases. As a result of this 1 - exp (-lamda $x$ Granularity) increases. Hence the probability that a node can fail in a particular interval increases. So, nodes failed increases as MTTF decreases. Hence throughput decreased as number of failed nodes increased. 


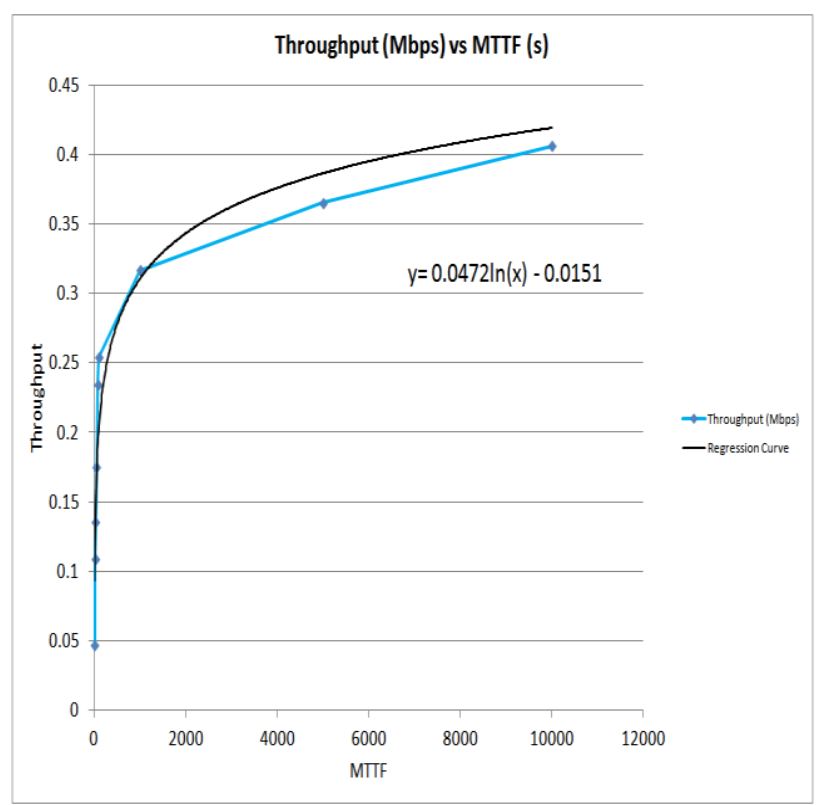

Figure 4. Throughput for Different Mean Time to Failures (MTTF's)

All the routes to destination might not be available due to node failures. Also packets have to be routed through a different route when some nodes are failed. Due to all these problems the throughput decreases with increase in number of failed nodes.

Throughput $=0.0472 * \ln (\mathrm{MTTF})-0.0151$ is the approximate equation obtained for Throughput vs. Mean Time to Failure.

The graph in Figure 5 shows the gradual decrease in throughput with increase in number of failure nodes.

From Figure 6 it can be observed that there is very slow decrease in reliability till the number of failed nodes is 17 out of 20 . The reliability goes down only when all the nodes are failed.

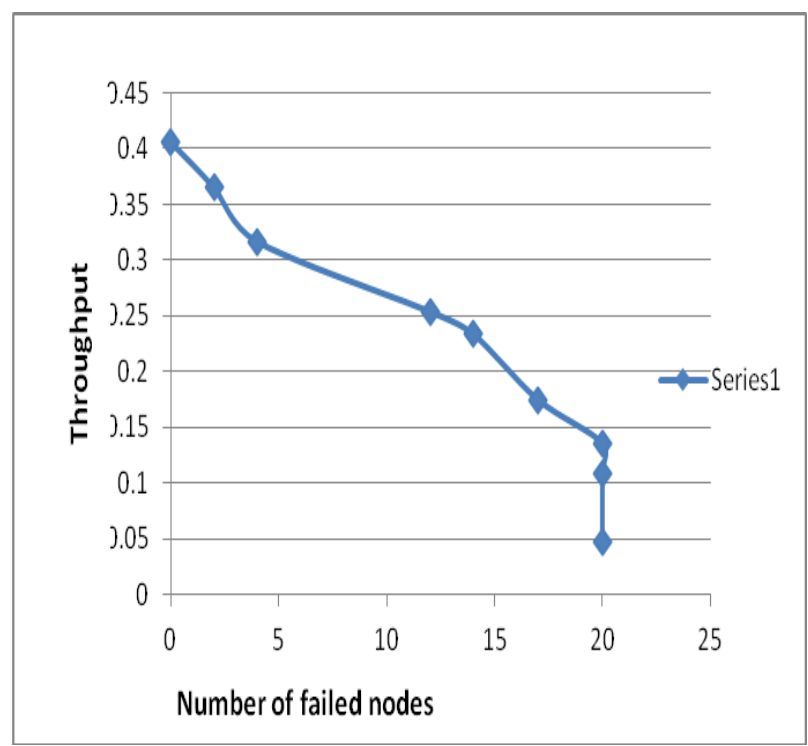

Figure 5. Number of Failed Nodes Vs Throughput 


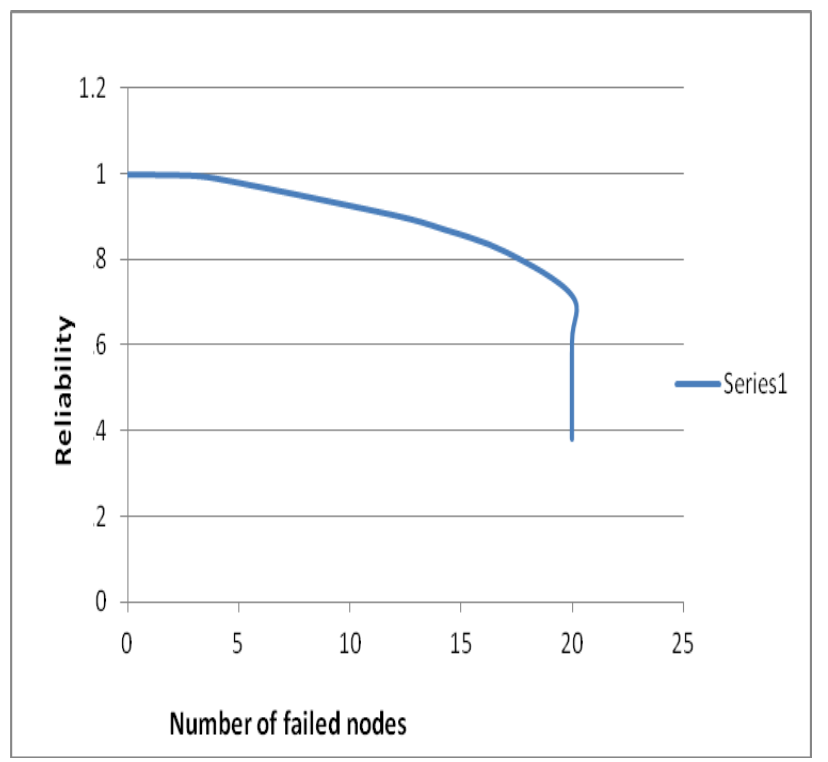

Figure 6. Number of Failed Nodes Vs Reliability

\section{Conclusion}

In this paper we have designed and developed a node failure model using Constant hazard model (CHM). To incorporate the node failure model, the NetSim is customized. We have setup simulation scenarios using customized NetSim and simulated the network using DSR protocol.

We find that all the routes to destination might not be available due to node failures. Also packets have to be routed through a different route when some nodes are failed. Due to all these problems the throughput decreases with increase in number of failed nodes.

The simulation approach outlined in this paper is also used for reliability evaluation of ad-hoc networks. We find that there is very less impact on reliability of node failure model until all the nodes get failed.

\section{Our Future Work will Focus on}

- A gap analysis is then carried out and strategies are planned to improve performance and reliability.

- Identification and implementation of improvement techniques.

\section{References}

[1] Min Xie, Yuan-Shun Dai, and Kim-Leng Poh, a book on "Computing System Reliability Models and Analysis", ISBN 0-306-48496-X, copyright 2004.

[2] Stephen Mueller, Rose P. Tsang, and Dipak Ghosal, "Multipath Routing in Mobile Ad Hoc Networks: Issues and Challenges", Department of Computer Science, University of California, Davis, CA 95616, Sandia National Laboratories, Livermore, CA 94551.

[3] Dongyan Chen,Sachin Garg, and Kishor S. Trivedi, "Network Survivability Performance Evaluation: A Quantitative Approach with Applications in Wireless Adhoc Networks", MSWiM'02, Atlanta, Georgia, USA., Copyright 2002 ACM 158113-6102/02/0009, (2002) September 28.

[4] N. Beikmahdavi, A. Akbari and F. Akbari, "Survey of Fault Recovery in Wireless Sensor Networks", International Journal of Latest Trends in Computing (E-ISSN: 2045-5364) 9, vol. 2, no. 1, (2001).

[5] P. Xiao and D. Liu, "Evaluating the Reliability of Large-Scale Heterogeneous Grid Computing Systems in Dynamic Workload Environments", International Journal of Future Generation Communication and Networking, vol. 6, no. 2, (2013) April.

[6] S. Kharbash and W. Wang, "Computing Two-Terminal Reliability in Mobile Ad hoc Networks", Department of Electrical and Computer Engineering, North Carolina State University, Raleigh, NC 27695. 
[7] T. Oliveira and F. Greve, "The Node Reliability Approach to Broadcasting in Manets: Raising Reliability with Low End-to-End Delay", Computer Science Department (DCC), Mechatronics Graduate Program, Federal University of Bahia, Salvador BA 40.170-110, Brazil.

[8] D. Pathirana, "RODMRP - Resilient On Demand Multicast Routing Protocol", A Thesis Submitted to the Faculty of the Rochester, Institute Of Technology, Computer Science, (2007) February.

[9] R. Leung, J. Liu, E. Poon, A.-L. Charles Chan and B. Li, "MP-DSR: A QoS-aware Multi-path Dynamic Source Routing Protocol for Wireless Ad-Hoc Networks", Department of Electrical and Computer Engineering, University of Toronto.

[10] S. Garg and K. S. Trivedi, "Network Survivability Performance Evaluation: A Quantitative Approach with Applications in Wireless Adhoc Networks", Dongyan Chen Center for Advanced Computing and Communications (CACC), Electrical and Computer Engineering Department, Duke University Durham, NC 277080294.

[11] M. Ali Cherif, M. Kamel Feraoun and S. Boukli Hacene, "Enhanced Predictive Preemptive Multipath Routing Protocol for Mobile Ad hoc Network", Computer Science Department, Sidi Bel Abbes University, Algeria, IEEE CommSoft E-Letters, vol. 2, no. 1, (2013) May.

[12] M. Conti, E. Gregori and G. Maselli, "Improving the performability of data transfer in mobile ad hoc networks", IIT Institute, CNR, Via G. Moruzzi 1, 56124 Pisa, Italy.

[13] K. Vinoth Kumar and S.Karthikeyan, "Multihop Energy Efficient Reliable and Fault Tolerant Routing Protocol for Wireless Sensor Networks", International Journal of Emerging Technology and Advanced Engineering, Website: www.ijetae.com, vol. 3, no. 2, (2013) February.

[14] E. W. Vollset and P. D. Ezhilchelvan, "Scribble: an Efficient Reliable Manycast protocol for Ad-hoc Networks", School of Computing Science University of Newcastle, UK.

[15] K. Erciyes, O. Dagdeviren, D. Cokuslu and D. Ozsoyelleryy, "Graph theoretic clustering algorithms in mobile ad hoc Networks and wireless sensor networks, Survey", Appl. Comput. Math., vol. 6, no. 2, (2007), pp. 162-180.

[16] M. Caleffi, G. Ferraiuolo and L. Paura, "A Reliability-based Framework for Multi-path Routing Analysis in Mobile Ad-Hoc Networks", Copyright (C) 200x, Inderscience Enterprises Ltd.

[17] S. Basagni, "Distributed Clustering for Ad Hoc Networks", Center for Advanced Telecommunications Systems and Services (CATSS), Erik Jonsson, School of Engineering and Computer Science EC33, The University of Texas at Dallas, 2601.

[18] J. L. Cook, P. Arsenal and J. Emmanuel Ramirez-Marquez, Stevens Institute of Technology, "Recent Research on the Reliability Analysis Methods for Mobile Ad-hoc Networks", Systems Research Forum, vol. 2, (2007).

[19] J. Wu, "Reliable Routing Protocols for Dynamic Wireless Ad Hoc and Sensor Networks", Ph.D. thesis, Enschede, the Netherlands, Copyright (2007).

[20] F. Erbas, K. Kyamakya and K. Jobmann, "On the Use of Position Information of Nodes in Mobile Ad hoc Networks", University of Hanover, Institute for Communications Engineering (IANT) Appelstrasse 9a, 30167 Hanover, Germany, proceedings of the 1st workshop on positioning, navigation and communication (WPNC'04).

[21] S. Gilbert, "Virtual Infrastructure for Wireless Ad Hoc Networks", Ph.D. thesis, Department of Electrical Engineering and Computer Science, Massachusetts Institute of Technology, (2007) August.

[22] L. Albini, A. Caruso, S. Chessa and P. Maestrini, "Reliable Routing in Wireless Ad Hoc Networks", University of Pisa, Via Buonarroti 2, 56127 Pisa, Italy.

[23] V. Vetri Selvi and R. Parthasarathi, "Mobile Ad Hoc Grid Architecture Using A Trace Based Mobility Model", Ubiquitous Computing and Communication Journal, www.ubicc.org, vol. 2.

[24] K.-H. Chen, C.-R. Dow, S.-C. Chen, Y.-S. Lee and S.-F. Hwang, "HarpiaGrid: A Geography-Aware Grid-based Routing Protocol for Vehicular Ad Hoc networks", Journal of Information Science and Engineering, vol. 26, (2010), pp. 817-832.

[25] R. Lent and J. Barria, "Towards Reliable Mobile Ad Hoc Networks", Imperial College London United Kingdom, www.intechopen.com.

[26] M. Caleffi, G. Ferraiuolo and L. Paura, "On Reliability of Dynamic Addressing Routing Protocols in Mobile Ad Hoc Networks", University of Naples "Federico II" via Claudio 21, Naples, 80125, Italy.

[27] J. Luo Patrick Th. and E. Jean-Pierre Hubaux, "Probabilistic Reliable Multicast in Ad Hoc Networks", Swiss Federal Institute of Technology (EPFL), CH-1015 Lausanne, Switzerland.

[28] A. Vishal Mundu and S. Gupta, "The application of system level diagnosis approaches for fault detection and its implementation in Ad-hoc networks", A thesis report Computer Science, NIT, India.

[29] E. Wiik Vollset, "Design and Evaluation of Crash Tolerant Protocols for Mobile Ad- hoc Networks", Thesis, Degree of Doctor of Philosophy, (Defended (2005) September 30).

[30] S. Mehta, Mst. Najnin Sulatan, H. Kabir, N. Ullah and K. S. Kwak, "Network and System Simulation Tools for Next Generation Networks: A Case Study", Inha University, Korea, www.intechopen.com.

[31] F. Li, A. Srinivasan, M. Lu and J. Wu, "Uncertainty Mitigation for Utility-Oriented Routing in Wireless Ad Hoc Networks", Department of Computer Science and Engineering, Florida Atlantic University Boca Raton, FL 33431.

[32] V. N. A. Naikan, a book on "Reliability Engineering and Life Testing", Copyright 2009 PHI Learning Private Ltd. New Delhi. 


\section{Authors}

Anil Choudhary completed his M.Sc. (Electronics and Communication) degree from Devi Ahilya Vishwavidyala, Indore, India and M. Tech. (Computer Science and Engineering) degree from Rajiv Gandhi University, Itanagar, India in 1996 and 2009, respectively. He is currently pursuing his research at North Eastern Regional Institute of Science and Technology ("NERIST" Deemed University under MHRD, Govt. of India), Itanagar, (Arunachal Pradesh). India and working as Head of the Department, Electrical and Electronics Engineering, Rajiv Gandhi Govt. Polytechnic, Arunachal Pradesh, India from 2010 and 2004, respectively. He was a Lecturer at the Faculty of Communication Engineering, Military College of Telecommunication Engineering (MCTE), Mhow, India from 2001 to 2002. He received the Broad Outlook Learner Teacher (BOLT) award instituted by Air India for the year 2007. He was also a recipient of the foreign fellowship under Technician Education - III project of MHRD, Govt. of India and attended advanced training in Electronics and Communication Engineering at Fulda, Germany. His research interests include MANET, Routing algorithm, and reliability.

O P Roy received the B. Tech. and M. Tech. in Electrical Engineering from State University and the Ph. D. in Electrical Engineering from Indian School of Mines (ISM) Dhanbad, India. He is presently working as an Associate Professor at the Department of Electrical Engineering, North Eastern Regional Institute of Science and Technology ("NERIST" Deemed University under MHRD, Govt. of India), Itanagar, (Arunachal Pradesh). His research interests are reliability engineering and Microprocessor based systems.

T. Tuithung received the B. Tech. (Computer Science and Engineering degree from North Eastern Regional Institute of Science and Technology (NERIST) Itanagar, (Arunachal Pradesh), and the $\mathrm{Ph}$. D. in Computer Science and Engineering from IIT Kharagpur, (WB), India. She is presently working as an Associate Professor at the Department of Computer Science and Engineering, North Eastern Regional Institute of Science and Technology ("NERIST" Deemed University under MHRD, Govt. of India), Itanagar, (Arunachal Pradesh). 